\title{
Preparation and Intrinsic Viscosity of Poly- ( $N$-methyl-2-vinylpyridinium chloride) with Narrow Molecular Weight Distributions
}

\author{
Masayoshi Yamaguchi, Yoshikazu Yamaguchi,* Yushu Matsushita, \\ and Ichiro NoDA \\ Department of synthetic Chemistry, Nagoya University, \\ Furo-cho, Chikasa-ku, Nagoya 464-01, Japan
}

(Received May 16, 1990)

\begin{abstract}
Anionically polymerized poly(2-vinylpyridine) was successfully quaternized with dimethyl sulfate in dimethylformamide at room temperature without degradation and coloring to prepare poly $(N$-methyl-2-vinylpyridinum chloride) (PMVP-C1) with narrow molecular weight distributions. It was found that the intrinsic viscosity of $\mathrm{PMVP}-\mathrm{Cl}$ in $\mathrm{NaCl}$ solutions agrees with the Stockmayer-Fixman equation except for high molecular weight samples at low ionic strengths, and the cubic of the expansion factor due to electrostatic repulsion obtained from intrinsic viscosity $\alpha_{\eta}^{3}$ is expressed as a linear function of $\left(M / C_{\mathrm{s}}\right)^{1 / 2}$ as reported for poly(sodium acrylate).

KEY WORDS Poly $(N$-methyl-2-vinylpyridinium chloride) / Polyelectrolyte

/ Quaternization / Narrow Molecular Weight Distribution / Intrinsic Viscosity

/ Expansion Factor /
\end{abstract}

In studying the physical properties of polymers at the molecular level, it is important to use polymer samples with narrow molecular weight distributions. This is the case for the study on macromolecular characteristics, such as intrinsic viscosity of polyelectrolytes as well.

Some polyelectrolyte samples with narrow molecular weight distributions have been prepared by an anionic polymerization. Kitano et $a l .^{1}$ reported that poly(acrylic acid) with narrow molecular weight distributions can be prepared by the hydrolysis of anionically polymerized poly(tert-butylacrylate), but the preparation of the low molecular weight sample with narrow molecular weight distributions is not easy, because the propagation in the polymerization of tert-butylacrylate is faster than the initiation. According to Higo et al., ${ }^{2}$ on the other hand, it is difficult to prepare poly(4-vinylbenzyltrimethylammonium chlor- ide) (PVBTMA-Cl) with high molecular weights because of the difficulty in eliminating impurities in 4-vinylbenzyldimethylamine, which is anionically polymerized. Poly(styrenesulfonic acid) with narrow molecular weight distributions can be prepared by the sulfonation of anionically polymerized polystyrene, but it is also not easy to obtain high molecular weight samples since there are possibility of crosslinking and degradation during sulfonation. ${ }^{3}$ As reported previously, ${ }^{4}$ poly(2-vinylpyridine) (P2VP) with narrow molecular weight distributions can be prepared over a wide range of molecular weight by an anionic polymerization. Thus, it is expected to obtain a desirable polyelectrolyte sample for viscosity study if P2VP is quaternized without degradation.

It is well-known that the intrinsic viscosity [ $\eta]$ of polyelectrolytes remarkably increases with decreasing concentration of added salt

* Present address: Japan Synthetic Rubber Co., Ltd., 100, Kawajiri-cho, Yokkaichi, Mie 510, Japan. 
$C_{\mathrm{s}}$, and the increase in $[\eta]$ is ascribed to expansion of the polyion chain due to electrostatic repulsion between charged segments apart on the polyion chain, or electrostatic excluded volume effect.

We reported that $[\eta]$ of poly(sodium acrylate)s ( $\mathrm{PNaA})$, prepared by fractionation, in the presence of added salt is in good agreement with the Stockmayer-Fixman equation. ${ }^{5,6}$

$$
[\eta] / M^{1 / 2}=K_{0}+0.51 \Phi_{0} B M^{1 / 2}
$$

where $K_{0}=\Phi_{0}\left(\left\langle R^{2}\right\rangle_{0} / M\right)^{3 / 2}, B=\beta / m_{\mathrm{s}}{ }^{2}, \Phi_{0}$ is the Flory viscosity coefficient, $M$ and $\left\langle R^{2}\right\rangle_{0}$ is the molecular weight and unperturbed mean square end-to-end distrance of polymer, respectively, and $\beta$ and $m_{\mathrm{s}}$ are the excluded volume and molecular weight of segment, respectively. This equation is transformed from the third-power law of the expansion factor for viscosity $\alpha_{\eta}{ }^{6}$

$$
[\eta] /[\eta]_{0} \equiv \alpha_{\eta}^{3}=1+1.55 z
$$

where $[\eta]_{0}\left(=K_{0} M^{1 / 2}\right)$ is the intrinsic viscosity at an unperturbed state, and $z=(3 / 2 \pi)^{3 / 2} B /$ $\left(\left\langle R^{2}\right\rangle_{0} / M\right)^{-3 / 2} M^{1 / 2}$. Later, 1.06 was reported in place of 1.55 for the front of $z .^{7}$

On the other hand, it is well established that $[\eta]$ of non-ionic polymers deviates from eq 1 and essentially follows the fifth-power law of Flory type such as eq 3 when the excluded volume effect is high. ${ }^{8,9}$

$$
\alpha_{\eta}^{5}-\alpha_{\eta}^{3}=k_{5} z
$$

where $k_{5}$ is a numerical constant.

Since the dependence of $\alpha_{\eta}$ on the excluded volume effect for polyelectrolyte appears to be different from that for non-ionic polymer, it is worth while to accumulate viscosity data of well-defined polyelectrolyte.

In this work, therefore, we examine the conditions of quaternization of P2VP to prepare poly $(N$-methyl-2-vinylpyridinium chloride) (PMVP-C1) with narrow molecular weight distributions and study $[\eta]$ of PMVP-Cl in $\mathrm{NaCl}$ solutions in terms of molecular weight and concentration of added salt $C_{\mathrm{s}}$.

\section{EXPERIMENTAL}

\section{Quaternization of Poly(2-vinylpyridine)}

An anionic polymerization of 2-vinylpyridine was carried out in tetrahydrofuran (THF) with cumyl potassium as an initiator in vacuo at $-78^{\circ} \mathrm{C}$, as reported previously. ${ }^{4}$

The conditions of quaternization were studied using various kinds of quaternizing reagents at various reaction times and temperatures. The experimental conditions are listed in Table I.

P2VP dissolved in polar solvents was reacted with an excess amount of quaternizing reagents under mild stirring. The polar solvents used here were dimethylsulfoxide (DMSO) and dimethylformamide (DMF), which dissolve P2VP, quaternizing reagents and quaternized samples so that the reaction occurs in a homogeneous solution. After a certain reaction time the reaction product was precipitated and washed with acetone. Then, it was dissolved in $1 \mathrm{M} \mathrm{NaCl}$ solution, and the polymer solution was dialyzed against $1 \mathrm{M} \mathrm{NaCl}$ solution so that the quaternized polymer was transformed to the chloride form (PMVP-C1).

\section{Characterization of Poly(N-methyl-2-vinylpyr-} idinium chloride)

Number-averaged molecular weights $M_{n}$ of P2VP and PMVP-C1 were determined by osmometry using a Hewlett-Packard High Speed Membrane Osmometer Type-502 in THF for P2VP and in $0.1 \mathrm{M} \mathrm{NaCl}$ solutions for PMVP-C1 at $25^{\circ} \mathrm{C}$. Weight-avaraged molecular weights $M_{w}$ were determined by light scattering with a Fica 50 Automatic Light Scattering Photometer in benzene for P2VP and in $0.1 \mathrm{M} \mathrm{NaCl}$ solutions for $\mathrm{PMVP}-\mathrm{Cl}$ at $25^{\circ} \mathrm{C}$. The wave length used was $436 \mathrm{~nm}$. Optical purification was carried out by filtration using $0.4 \mu \mathrm{m}$ Millipore filter for P2VP and $0.4 \mu \mathrm{m}$ Nucleopore filter for PMVP-Cl. The refractive index increment $\partial n / \partial c$ of 
PMVP-C1 in $0.1 \mathrm{M} \mathrm{NaCl}$ solutions determined with a Shimadzu differential refractometer at $25^{\circ} \mathrm{C}$ is $0.208 \mathrm{mlg}^{-1}$, which is almost independent of the degree of quaternization for the samples used here within experimental errors, and $\partial n / \partial c$ of P2VP in benzene used is $0.107 \mathrm{mlg}^{-1}$ reported by Arichi et al. ${ }^{10}$

GPC elution curves of PMVP-Cl were measured with a Toso High Speed Chromatograph HLC-802UR using Toso High Resolution Columns PW5000 and PW2000. The eluting solvent used was $0.3 \mathrm{M} \mathrm{Na}_{2} \mathrm{SO}_{4}$ solution containing $10 \% \mathrm{CH}_{3} \mathrm{COOH}$, which was added to avoid adsorption of the sample on the gel matrix. ${ }^{11}$ The degree of quaternization was determined by neutralization titration after PMVP-C1 was transformed to its hydroxide form by passing through an ion-exchange resin column.

Polymer concentrations of PMVP-C1 solutions were determined by measuring UV absorption at $267 \mathrm{~nm}$ with a Shimadzu UVVisible Recording Spectrophotometer UV-240. The caribration curve was made by using samples freeze-dried from salt-free solutions and dried with $\mathrm{CaCl}_{2}$ in vacuo for 30 days till the weights became constant.

\section{Intrinsic Viscosity Measurements}

Viscosity measurement was carried out in $0.5,0.1$ and $0.01 \mathrm{M} \mathrm{NaCl}$ solutions at $25^{\circ} \mathrm{C}$ with a viscometer of modified Ubbelohde type with 4 bulbs. The zero-shear viscosity was evaluated by extrapolating the inverse of observed relative viscosity $1 / \eta_{\text {rel }}^{\text {obs }}$, which was plotted against the square of head $h^{2}$, to the true one $1 / \eta_{\text {rel }}$ at the zero head, ${ }^{12}$ when the rate-shear dependence was observed, and the intrinsic viscosity was determined from the common intercept in the plots of $\eta_{\mathrm{sp}} / c v s . c$, $\ln \left(\eta_{\text {rel }}\right) / c$ vs. $c$ and $\left\{2\left(\eta_{\text {sp }}-\ln \eta_{\text {rel }}\right)\right\}^{1 / 2} / c$ vs. $c .^{13}$

\section{RESULTS AND DISCUSSION}

\section{Quaternization}

Experimental results of quaternization are summarized in Table I. When methyl iodide and ethyl bromide were used as quaternizing reagents in DMSO,${ }^{14}$ the quaternized samples were colored dark brown and brown, respectively. The colored samples are not suitable for measurements and also suggest that unfavorable

Table I. Quaternization of poly(2-vinylpyridine)s

\begin{tabular}{|c|c|c|c|c|c|c|c|c|}
\hline \multirow{2}{*}{$\begin{array}{l}\text { Exptl. } \\
\text { No. }\end{array}$} & \multirow{2}{*}{$\begin{array}{c}M_{w} \\
\left(\times 10^{3}\right)\end{array}$} & \multirow{2}{*}{$\begin{array}{l}\text { Quaternizing } \\
\text { reagent }\end{array}$} & \multirow{2}{*}{ Solvent } & \multirow{2}{*}{$\begin{array}{l}\text { Reaction } \\
\text { time }\end{array}$} & \multirow{2}{*}{$\frac{\text { Temperature }}{\mathrm{C}^{\circ}}$} & \multirow{2}{*}{ D.Q. ${ }^{\mathrm{a}}$} & \multirow{2}{*}{ M.W.D. ${ }^{b}$} & \multirow{2}{*}{ Color } \\
\hline & & & & & & & & \\
\hline 1 & 670 & Methyl iodide & DMSO & $8 \mathrm{~h}$ & 135 & - & - & Brown \\
\hline 2 & & & & $12 \mathrm{~h}$ & 50 & - & - & Brown \\
\hline 3 & & Ethyl bromide & DMSO & $72 \mathrm{~h}$ & 49 & 22 & - & Yellow \\
\hline 4 & & Methyl- $(p)-$ & & $72 \mathrm{~h}$ & 65 & 44 & - & Colorless \\
\hline 5 & & toluene- & DMSO & $72 \mathrm{~h}$ & 100 & 46 & - & Colorless \\
\hline 6 & & sulfonate & & 1 week & 55 & 36 & - & Colorless \\
\hline 7 & & Dimethyl & DMF & 10 days & R.T. & 81.6 & $\mathrm{~S}$ & Colorless \\
\hline 8 & & sulfate & & 30 days & R.T. & 100 & B & Colorless \\
\hline 9 & 2000 & & & 10 days & R.T. & - & D & Colorless \\
\hline 10 & 100 & & & $24 \mathrm{~h}$ & R.T. & 89.7 & $\mathrm{~S}$ & Colorless \\
\hline 11 & 452 & & & $24 \mathrm{~h}$ & R.T. & 87.3 & $\mathrm{~S}$ & Colorless \\
\hline 12 & 720 & & & $24 \mathrm{~h}$ & R.T. & 85.3 & $\mathrm{~S}$ & Colorless \\
\hline 13 & 1950 & & & $24 \mathrm{~h}$ & R.T. & 81.8 & $\mathrm{~S}$ & Colorless \\
\hline
\end{tabular}

a D.Q., represents the degree of quaternization.

b M.W.D., represents the molecular weight distribution. The letters S, D and B denote the sharp, double and broad distributions, respectively. 
side reactions might occur. The quaternization of P2VP appears to be slower than that of poly(4-vinylpyridine). ${ }^{15}$ This may be due to the difference between steric hindrances of their pyridine units. Since coloring may be due to halogen, methyl- $(p)$-toluenesulfonate, which does not contain halogen, was used for quaternization. In this case, the degree of quaternization was below $50 \%$ even if the temperature was raised or the reaction time became longer as shown in Table I. To increase the degree of quaternization, therefore, dimethyl sulfate, which is a vigorous reagent for methylation was employed in DMF suited for the reagent. When quaternization was carried out for $24 \mathrm{~h}$ at a room temperature $\left(\sim 25^{\circ} \mathrm{C}\right)$, the degree of quaternization was higher than $80 \%$ as shown in Table I. If the reaction was continued for 30 days, the degree of quaternization increased up to $100 \%$, but the sample was degradated as shown by the GPC elution curves in Figure 1. The high molecular weight sample was degradated even if the reaction time was 10 days, while the low molecular weight sample was not, as shown in Figure 1 and Table I.

\section{Preparation and Characterization of $\operatorname{Poly}(N-$} methyl-2-vinylpyridinium chloride)s

On the basis of the quaternization study mentioned above, four P2VPs were quaternized with dimethyl sulfate in DMF at a room temperature for 24 hours. The molecular char- acteristics of PMVP-Cls together with those of the corresponding P2VPs are shown in Table II. The $M_{w} / M_{n}$ of PMVP-Cls were evaluated from the GPC elution curves using the calibration curve between the elution volume and molecular weight, which was determined by trial and error until the molecular weights measured and calculated from the calibration curve agreed with each other. The broadening of GPC elution curves due to the axial dispersion of solute in columns was corrected by using the $M_{w} / M_{n}$ values of standard pullulan of Showa Denko Co. Ltd.
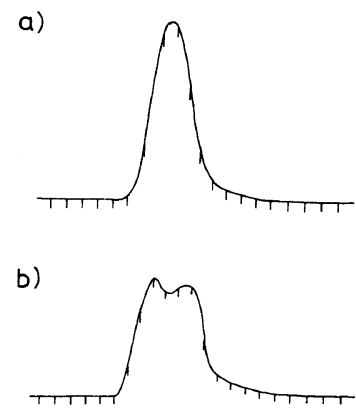

c)

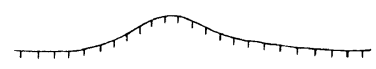

Figure 1. GPC elution curves of samples quaternized under various conditions: a) exptl. No. 11 in Table I $\left(M_{w}=452 \times 10^{3}, 24 \mathrm{~h}\right)$; b) exptl. No. $9\left(M_{w}=2 \times 10^{6}\right.$, 10 days); c) exptl. No. $8\left(M_{w}=670 \times 10^{3}, 30\right.$ days $)$.

Table II. Molecular characteristics of $\operatorname{poly}(N$-methyl-2-vinylpyridinium chloride) (PMVP-Cl) and poly(2-vinylpyridine) (P2VP)

\begin{tabular}{|c|c|c|c|c|c|c|c|c|}
\hline \multirow{2}{*}{$\begin{array}{l}\text { Sample } \\
\text { code }\end{array}$} & \multicolumn{4}{|c|}{ PMVP-Cl (I) } & \multicolumn{3}{|c|}{ P2VP (II) } & \multirow{2}{*}{ D.P. II/D.P. I' } \\
\hline & $M_{w} \times 10^{-3}$ & $M_{w} / M_{n}$ & D.Q. ${ }^{\mathrm{a}}$ & D.P. ${ }^{b}$ & $M_{w} \times 10^{-3}$ & $\mathrm{M}_{w} / M_{n}$ & D.P. ${ }^{b}$ & \\
\hline MVPK10 & 140 & 1.04 & 89.7 & 930 & 98 & 1.04 & 930 & 1.00 \\
\hline MVPK11 & 694 & 1.07 & 87.3 & 4670 & 452 & 1.04 & 4300 & 1.09 \\
\hline MVPK12 & 1100 & 1.09 & 85.3 & 7430 & 720 & 1.07 & 6860 & 1.08 \\
\hline MVPK13 & 2740 & 1.13 & 81.8 & 18800 & 1950 & 1.15 & 18500 & 1.02 \\
\hline
\end{tabular}

a D. Q., represents the degree of quaternization.

b D. P., represents the degree of polymerization.

c The ratio of the degree of polymerization of P2VP to that of PM2VP-C1. 
Since the molecular weights of PMVP-Cls are in good agreement with the values calculated from the molecular weights of the corresponding P2VPs and the degree of quaternization within the experimental errors, and the both $M_{w} / M_{n}$ values are almost equal, we may conclude that no degradation of polymer chain occurred during quaternization, and the PMVP-Cl samples as well as P2VP samples have relatively narrow molecular weight distributions. The degree of quaternization was $80-90 \%$, slightly depending on the molecular weight. Fortunately, solution properties of polyelectrolytes are not very much affected by charge density if it is higher than $80 \%$ as shown in a previous work for poly(sodium acrylate)s. ${ }^{5}$

Intrinsic Viscosity of Poly( $N$-methyl-2-vinylpyridinium chloride)s in the Presence of Added Salt

Double logarithmic polts of $[\eta]$ of PMVP$\mathrm{Cl}$ vs. $M_{w}$ in $0.01,0.1$ and $0.5 \mathrm{M} \mathrm{NaCl}$ solutions are shown in Figure 2. The MarkHouwink-Sakurada equations determined by the least-squares method are given by

$$
\begin{array}{cc}
{[\eta]=7.86 \times 10^{-5} M_{w}{ }^{0.86}} & \left(C_{\mathrm{s}}=0.01 \mathrm{M}\right) \\
{[\eta]=8.40 \times 10^{-5} M_{w}{ }^{0.77}} & \left(C_{\mathrm{s}}=0.1 \mathrm{M}\right) \\
{[\eta]=2.68 \times 10^{-4} M_{w}{ }^{0.63}} & \left(C_{\mathrm{s}}=0.5 \mathrm{M}\right)
\end{array}
$$

Figure 3 shows the Stockmayer-Fixman plots for PMVP-Cl at various $\mathrm{NaCl}$ concentrations. Apparantly, $[\eta] / M^{1 / 2}$ of PMVP-Cl agrees with the Stockmayer-Fixman equation except for high molecular weight samples at low ionic strengths. From the intercept at the ordinate we have $K_{0}=0.95( \pm 0.05) \times 10^{-3}$. Using $\Phi_{0}=2.5 \times 10^{21}$, we have $\left\langle R^{2}\right\rangle_{0} / M=$ $5.2( \pm 0.2) \times 10^{-17} \mathrm{~cm}^{2} \mathrm{~g}^{-1}$ and $2.8( \pm 0.1)$ for the characteristic ratio $\sigma$. The $\sigma$ value is comparable to those of polystyrene (2.3), P2VP (2.3), PNaA (2.4) and PVBTMA-Cl (3.2). Thus, $\mathrm{PMVP}-\mathrm{Cl}$ is a typical flexible polyelectrolyte.

The expansion factor for viscosity $\alpha_{\eta}$ is evaluated from $\alpha_{\eta}^{3}\left(=[\eta] / K_{0} M^{1 / 2}\right)$ by using the

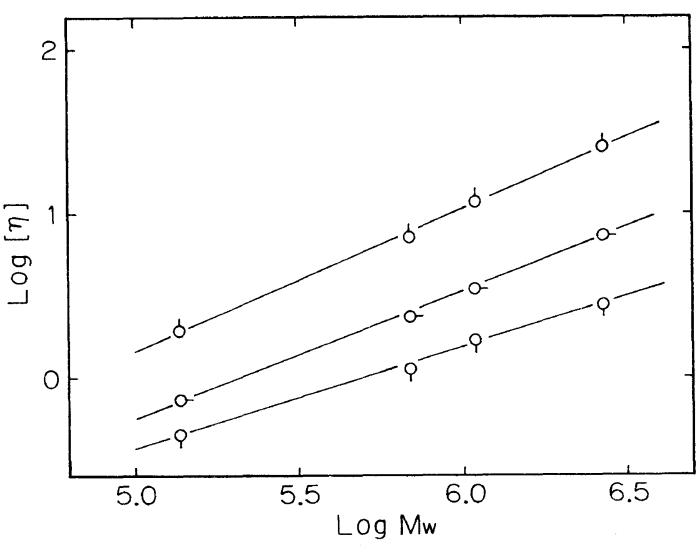

Figure 2. Double logarithmic plots of $[\eta] v s . M_{w}$ for PMVP-Cl in $\mathrm{NaCl}$ solutions: (o) $C_{\mathrm{s}}=0.01 \mathrm{M} \mathrm{NaCl}$; (o-) $C_{\mathrm{s}}=0.1 \mathrm{M} \mathrm{NaCl} ;$ ( () $C_{\mathrm{s}}=0.5 \mathrm{M} \mathrm{NaCl}$.

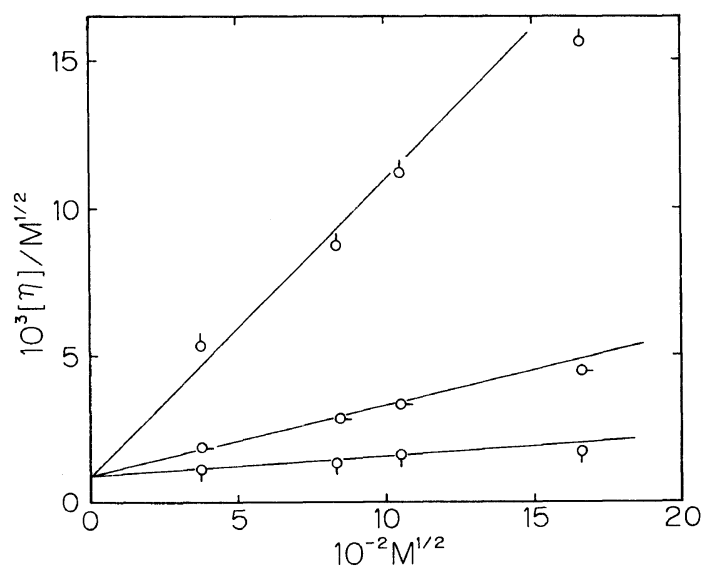

Figure 3. Stockmayer--Fixman plots of PMVP-Cl in $\mathrm{NaCl}$ solutions. The symbols are the same as in Figure 2.

observed $K_{0}$ value, since the unperturbed dimension of polyelectrolyte can be assumed to be almost independent of the concentration of added salt at least at high ionic strengths. ${ }^{16}$ The excluded volume of charged segment $\beta$ can be assumed to consist of electrostatic $\left(\beta_{\mathrm{el}}\right)$ and non-electrostatic ones $\left(\beta_{0}\right) ; \beta=\beta_{\mathrm{el}}+\beta_{0}$. Since the electrostatic repulsion is shielded and hence, $\beta_{\mathrm{el}}=0$ at infinite ionic strength, the electrostatic expansion factor $\alpha_{\eta_{\mathrm{e}}}$ due to $\beta_{\mathrm{el}}$ can be evaluated by ${ }^{5}$

$$
\alpha_{\eta_{\mathrm{e}}}^{3}=\alpha_{\eta}^{3}-\lim _{c_{\mathrm{s}} \rightarrow 0}\left(\alpha_{\eta}^{3}-1\right)
$$




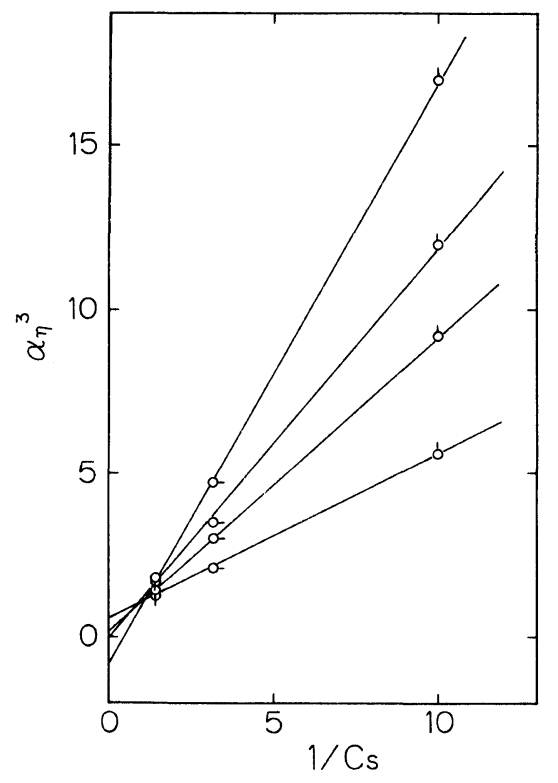

Figure 4. Plots of $\alpha_{\eta}^{3} v s .1 / C_{\mathrm{s}}{ }^{1 / 2}$. The molecular weights decrease from top to bottom.

Since the well-known linear relationship between $\alpha_{\eta}^{3}$ and $C_{\mathrm{s}}^{-1 / 25}$ is observed also for PMVP-Cl as shown in Figure $4, \mathrm{C}_{\mathrm{s}} \rightarrow \infty$ can be obtained as $C_{\mathrm{s}}^{-1 / 2}=0$.

A combination of the linear relationship between $\alpha_{\eta}^{3}$ and $C_{\mathrm{s}}{ }^{-1 / 2}$, and eq 2 suggests the following equation for $\alpha_{\eta_{e}}{ }^{5}$

$$
\begin{aligned}
& \alpha_{\eta_{\mathrm{e}}}^{3}-1=k_{3} z_{\mathrm{el}} \\
z_{\mathrm{el}}= & (3 / 2 \pi)^{3 / 2}\left(\beta_{\mathrm{el}}{ }^{\prime} / m_{\mathrm{s}}{ }^{2}\right) \\
& \left(\left\langle R^{2}\right\rangle_{0} / M\right)^{-3 / 2}\left(M / C_{\mathrm{s}}\right)^{1 / 2}
\end{aligned}
$$

where $z_{\mathrm{el}}$ is the electrostatic excluded volume parameter, $\beta_{\mathrm{el}}{ }^{\prime}=\beta_{\mathrm{el}} C_{\mathrm{s}}{ }^{1 / 2}$ and $k_{3}$ is a numerical constant.

Theoretically, $z_{\mathrm{el}}$ can be derived assuming the electrostatic excluded volume to be an interaction between charged cylinders. ${ }^{17,18}$ Taking into account ion-binding given by the theory of Manning, ${ }^{19}$ Odijk and Houwaart ${ }^{17}$ presented the theoretical equation of $\beta_{\mathrm{el}}$ as

$$
\beta_{\mathrm{e} 1}=2 \pi b^{2} / \kappa
$$

where $b$ is the statistical segment length of the polyion and $1 / \kappa$ is the Debye-Hückel screening

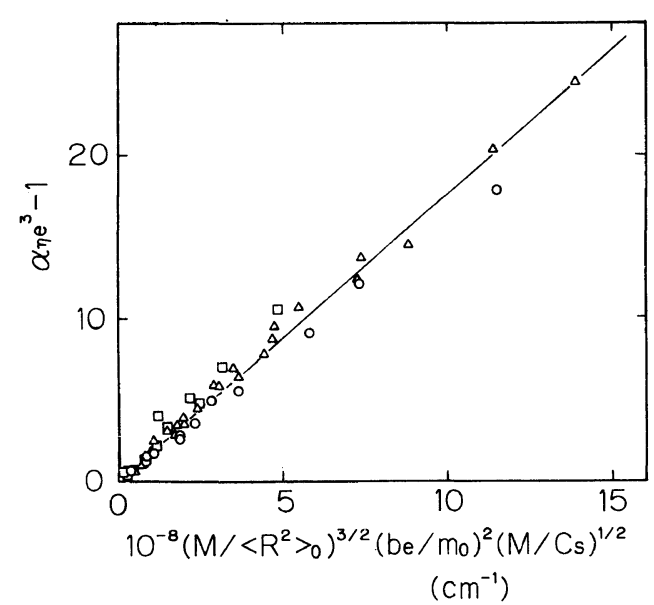

Figure 5. Plots of $\alpha_{\eta_{\mathrm{e}}}^{3}-1$ vs. $\left(M /\left\langle R^{2}\right\rangle_{0}\right)^{3 / 2}\left(b_{\mathrm{e}} / m_{0}\right)^{2}$ $\left(M / C_{\mathrm{s}}\right)^{1 / 2}$ Circles denote the present data, triangles denote the data of PNaA with $i=1.0$ (ref 5) and the squares denote the data of PVBTMA-Cl (ref 11).

length. Since Fixman and Skolnick ${ }^{18}$ presented almost the same equation as eq 6 , but they did not take into account ion-binding, we compared data with the theory of Odijk and Houwaart. ${ }^{17}$ Using eq 5 and 6 , and $1 / \kappa=3.043 \times 10^{-8} / C_{\mathrm{s}}{ }^{1 / 2} \mathrm{~cm}$ in $\mathrm{NaCl}$ solutions at $25^{\circ} \mathrm{C}$, where $C_{\mathrm{s}}$ is expressed in $\mathrm{M}$, we have

$$
\begin{aligned}
z_{\mathrm{el}}= & \left(2 \pi \times 3.403 \times 10^{-8}\right)(3 / 2 \pi)^{3 / 2}\left(b / m_{\mathrm{s}}\right)^{2} \\
& \left(\left\langle R^{2}\right\rangle_{0} / M\right)^{-3 / 2}\left(M / C_{\mathrm{s}}\right)^{1 / 2}
\end{aligned}
$$

Figure 5 shows plots of $\alpha_{\eta_{\mathrm{e}}}^{3}$ vs. $\left(b_{\mathrm{e}}\right)$ $\left.m_{0}\right)^{2}\left(\left\langle R^{2}\right\rangle_{0} / M\right)^{-3 / 2}\left(M / C_{\mathrm{s}}\right)^{1 / 2}$ for PMVP-C1 in various $\mathrm{NaCl}$ solutions, together with data of $\mathrm{PNaA}$ in $\mathrm{NaBr}$ solutions ${ }^{5}$ and PVBTMA-Cl in $\mathrm{NaCl}$ solutions. ${ }^{11}$ Here, we use $b_{\mathrm{e}} / m_{0}$ in place of $b / m_{\mathrm{s}}$ in eq 7 , where $b_{\mathrm{e}}$ and $m_{0}$ are the effective length and molecular weight of the monomer, respectively. In the treatment of the Kuhn statistical segment $b_{\mathrm{e}}=l \sin (\theta / 2)$, where $l$ is the length of monomer unit and $\theta$ is the bond angle. It is apparent that $\alpha_{\eta_{e}}^{3}$ is a linear and universal function of $\left(b_{\mathrm{e}} / m_{0}\right)^{2}$ $\left(\left\langle R^{2}\right\rangle_{0} M\right)^{-3 / 2}\left(M / C_{\mathrm{s}}\right)^{1 / 2}$ irrespective of the polyion species, though the experimental $z_{\text {el }}$ value is $3-5$ times larger than eq 7 depending on the $k_{3}$ value in eq $5 \mathrm{a}$. Therefore, we can conclude that the intrinsic viscosity of poly- 
electrolytes in the present ranges of molecular weight and concentration of added salt is explained by eq 5 .

Acknowledgment. This work was supported by a Grant-in Aid for Scientific Research (No. 63430019) from the Ministry of Education, Science, and Culture of Japan.

\section{REFERENCES}

1. T. Kitano, T. Fujimoto, and M. Nagasawa, Polym. J., 9, 153 (1977).

2. Y. Higo, H. Chosi, and M. Nagasawa, Polym. J., 12, 729 (1980).

3. M. Nagasawa and T. Fujimoto in "KobunshiJikkengaku," Vol. 13, The Society of Polymer Science, Japan, Ed., Kyoritsu Shuppan Co., Tokyo, 1980, Chapter 2.

4. Y. Matsushita, K. Shimizu, Y. Nakao, H. Choshi, I. Noda, and M. Nagawawa, Polym. J., 18, 361 (1986).

5. I. Noda, T. Tsuge, and M. Nagasawa, J. Phys. Chem., 74, 710 (1970).

6. W. H. Stockmayer and M. Fixman, J. Polym. Sci., C, 1, 137 (1963).
7. H. Yamakawa and G. Tanaka, J. Chem. Phys., 55, 3188 (1971).

8. P. J. Flory, "Principles of Polymer Chemistry," Cornell University Press, Ithaca, New York, N.Y., 1953.

9. H. Yamakawa, "Modern Theory of Polymer Solutions," Harper \& Row, Publishers, Inc., New York, N. Y., 1971.

10. S. Arichi, M. Yoshida, and Y. Ogawa, Bull. Chem. Soc. Jpn., 48, 1417 (1975).

11. Y. Higo, Y. Kato, M. Itoh, N. Kozuka, I. Noda, and M. Nagasawa, Polym. J., 14, 809 (1982).

12. R. M. Davis and W. B. Russel, Macromolecules, 20, 518 (1987).

13. S. H. Maron and R. B. Reznik, J. Polym. Sci. A-2, 7, 309 (1969).

14. E. Pefferkorn, Q. Tran, and R. Varoqui, J. Polym. Sci., Polym. Chem. Ed., 19, 27 (1981).

15. R. M. Fuoss, M. Watanabe, and B. D. Coleman, $J$. Polym. Sci., 48, 5 (1960).

16. Y. Muroga, I. Noda, and M. Nagasawa, Macromolecules, 18, 1576 (1985).

17. T. Odijk and A. C. Houwaart, J. Polym. Sci., Polym. Phys., 16, 627 (1978).

18. M. Fixman and J. Skolnick, Macromolecules, 11, 863 (1978).

19. G. S. Manning, J. Chem. Phys., 51, 924 (1969). 